\title{
Repair of perforation with Mineral trioxide aggregate (MTA)
}

\author{
Hariharavel VP ${ }^{1}$, Ashok Kumar A ${ }^{2}$, Sankar Annamalai ${ }^{3}$, Aravindhan $\mathrm{R}^{4}$, \\ Kavitha Ramar ${ }^{5}$ \\ ${ }^{I}$ (Department of Pedodontics and Preventive Dentistry, SRM KDC, India) \\ ${ }_{2}^{2}$ (Consultant Endodontist, Sri Ammaiappan Dental Clinic, India) \\ ${ }_{3}^{3}$ (Department of Pedodontics and Preventive Dentistry, SRM KDC, India) \\ ${ }^{4}$ (Department of Oral Pathology, SRM KDC, India) \\ ${ }_{5}^{5}$ (Department of Pedodontics and Preventive Dentistry, SRM KDC, India)
}

\begin{abstract}
Root perforations may occur due to pathological processes or treatment consequences. Such perforations are severe complications and are associated with dramatically compromised endodontic treatment outcomes, especially when bacterial infection is allowed to establish and if the seal between the endodontic space and periradicular tissue is not provided. A new known material, mineral trioxide aggregate (MTA) promotes a favorable environment for regeneration and has been successfully used for various endodontic surgeries, apexification and perforation procedures. This case report describes the management of perforation which occurred in the critical region of tooth.
\end{abstract}

Keywords: MTA, Perforation, Anterior tooth

\section{INTRODUCTION}

Endodontic mishaps are not very uncommon and in that perforations are no exception. According to Kvinnsland et al [1] 53\% of iatrogenic perforations occur during insertion of posts and the remaining $47 \%$ are induced during routine endodontic treatment. Root perforations which are induced, possess significant complications to endodontic treatment and if not detected and properly treated, the breakdown of the periodontium may ultimately lead to loss of the tooth.

Perforation is defined as mechanical or pathological communications between the root canal system and the external tooth surface [2]. The injury to the periodontium results in the development of inflammation, destruction of periodontal fibers, bone resorption, formation of granulomatous tissue, proliferation of epithelium, ultimately in the development of a periodontal pocket, mobility and finally loss of tooth. Anatomical variations are not anticipated during access preparation and as a result of misalignment of the bur with the long axis of the root, crowns of many teeth are frequently perforated. [3]

Various materials have been used in managing perforations, including zinc oxide-eugenol, amalgam, calcium hydroxide, IRM, super EBA, composite resin, glass ionomer and resin modified glass ionomer. The ideal material for treating radicular perforations should be nontoxic, non-absorbable, radiopaque, and bacteriostatic or bactericidal; it should also provide a seal against microleakage from the perforation [4]. Mineral trioxide aggregate (MTA) has all of these characteristics and has been applied with good outcomes in root-end surgery, direct pulpal coverage, apexification, radicular resorption, repair of lateral radicular and furcal perforation. [5]

Although several perforation cases have been reported, there are limited numbers of studies published on the use of MTA in perforation repair in the cervical region of tooth. This paper discusses one such encounter.

\section{CASE REPORT}

A 26 year old male patient with a noncontributory medical history reported to our clinic with chief complaint of pain in maxillary right central incisor. History revealed an attempt of root canal treatment procedure in some other dental clinic which was unsuccessful and hence was referred here. Clinical examination revealed tenderness on percussion in maxillary right central incisor, ulceration in the attached gingiva and periodontal pocket $(4 \mathrm{~mm})$ in the labial aspect. Radiographic examination revealed a $5 \mathrm{~mm}$ deficient radio opaque material, from the pulp chamber into the canal, suggestive of improper obturation with Gutta percha (GP) point [Fig. 1]. An iatrogenic root canal perforation in the labial aspect was suspected and was confirmed by intracanal exploration.

Different treatment options have been discussed with the patient. Since the patient was from a remote location and wanted the treatment in a single visit, root canal treatment of maxillary right central incisor along with surgical repair of the perforation with MTA was finalised.

After the administration of the local anesthetic (2\% Lignocaine with 1:100,000 epinephrine), root canal was renegotiated and proper biomechanical preparation and irrigation was done. Mucoperiosteal flap was raised 
from 12 to 22 region and a perforation of size $2 \mathrm{~mm}$ diameter was located which was cleaned with saline [Fig. 2]. The debris and granulation tissues were removed using k-file size 30 [Fig. 3]. GP point, size 60 was placed in the canal as a template to maintain the patency of the canal, and the perforation was sealed with white MTA (Angelus, Londrina, Brazil) mixed with sterile saline, as supplied by the manufacturer. The MTA was covered with a cotton pellet moistened with distilled water [Fig. 4]. After initial setting of MTA, the GP point was removed and obturated by lateral condensation technique without disturbing the MTA [Fig. 5]. Set of MTA was checked and the flap was closed, sutured and COE-PAK (GC America INC) was placed [Fig. 6].

At the 1 week follow-up, the patient was asymptomatic [Fig. 7]. Suture was removed and there was no sign of periodontal inflammation or pocket formation. Patient was advised crown for the treated tooth. The patient had conveyed that he was asymptomatic and comfortable, hence not reported for the sixth month follow up.

\section{DISCUSSION}

Perforation is an undesirable problem that may occur during root canal treatment, caries removal and post preparation. Time of repair, size of perforation and location of perforation are the factors which affects the prognosis of perforation treatment [6]. In any perforation case, if the situation is quickly addressed and treated immediately with suitable material, it ensures a positive prognosis. In our present case, even though the patient reported to the clinic very soon after the perforation and the size of the perforation was small, the challenge was the location of perforation which was in the 'critical zone' area. [7]

MTA was selected as the material of choice in the present case because of its good sealing ability, biocompatibility, bactericidal effect, radio-opacity and its successful use as root-end fillings material, in surgical repair, pulp capping, apexification, radicular resorption and perforations [5]. Two types of MTA are available, Grey and white. White MTA was preferred in this case since it is associated with the development of cementoblasts and keratinocytes [8], [9]; though both prove to be good in many perforation cases.

There were high chances of MTA getting extruded through the perforation to periodontal space which would cause periodontal pocket later. Confining the material only to the perforated region could be achieved only through flap elevation, hence surgical approach was decided. We preferred to place MTA first and obturation of GP later, since it was comfortable for eight handed dentistry to finish the procedure fast and without complications. Although the use of MTA has been suggested for several different endodontic treatments, literature reporting successful treatment outcomes in perforation repair is limited.

Another important factor in non-surgical as well as surgical perforation repair, is to achieve a fluidtight seal between the tooth and the repair material. So placing COE - PAK (periodontal dressing) for maintaining the seal and heal was planned as it provides an added advantage to achieve the tight seal and repair of periodontium at the cervical region.

\section{FIGURES AND TABLES}

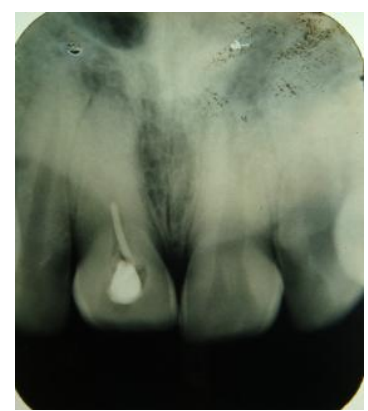

Figure 1 - Pre operative Radiograph

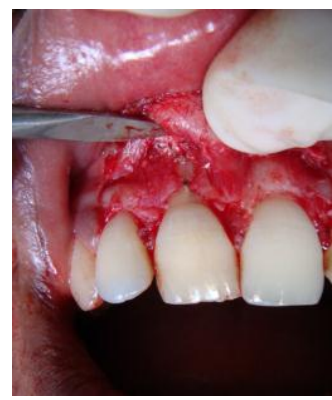


Figure 2 - Raised flap showing perforation

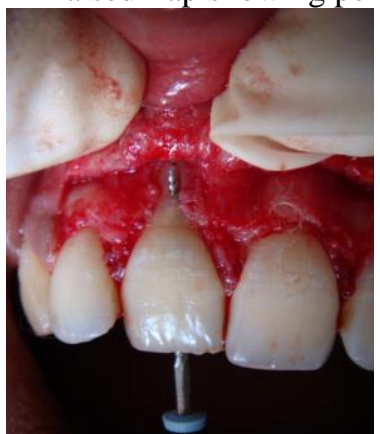

Figure 3 - Debris and granulation tissues are removed using K file

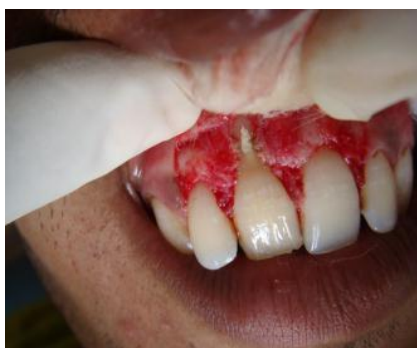

Figure 4 - Perforation repair with MTA

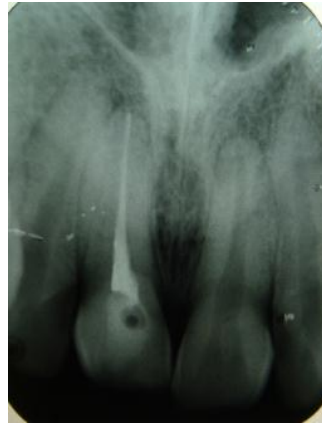

Figure 5 - Immediately after obturation

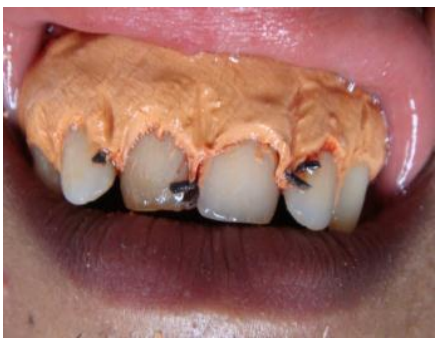

Figure 6 - Flap was sutured and closed with COE-PAK

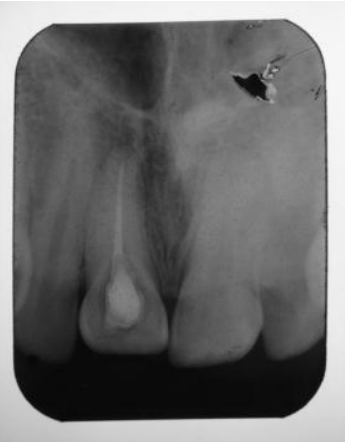

Figure 7 - At 1 week follow-up 
V.

CONCLUSION

Although the prognosis is generally better for smaller lesions, the location of perforation at critical area was a greater challenge, and successful use of MTA with the use of adjuvant periodontal dressing played key role in successful outcome of the treatment. MTA is a remarkable material in the hands of dentists to deal such endodontic mishaps successfully that are previously regarded as hopeless.

\section{REFERENCES}

[1] Kvinnsland I, Oswald RJ, Halse A, Gronningsaeter AG. A clinical and roentgenological study of 55 cases of root perforation. Int Endod J, 22, 1989, 75-84.

[2] Glossary of Endodontic Terms. American Association of Endodontists, (Chicago IL, 2003)

[3] Gutmann JL, Dumsha TC, Lovdahl PE. Problem Solving in Endodontics. (Mosby, 2006)

[4] De-Deus G, Reis C, Brandão C, Fidel S, Fidel RA. The ability of Portland cement, MTA, and MTA Bio to prevent through-andthrough fluid movement in repaired furcal perforations. J Endod, 33(11), 2007, 1374-7.

[5] Torabinejad M, Chivian N. Clinical application of mineral trioxide aggregate. J Endod, 25(3), 1999, $197-205$.

[6] Thomas Clauder, Su-Jung Shin. Repair of perforations with MTA: clinical applications and mechanisms of action. Endodontic Topics, 15, 2009, 32-55.

[7] Fuss Z, Trope M. Root perforations: classification and treatment choices based on prognostic factors. Endod Dent Traumatol, 12, 1996, 255-264.

[8] Oviir T, Pagoria D, Ibarra G, Geurtsen W. Effects of gray and white mineral trioxide aggregate on the proliferation of oral keratinocytes and cementoblasts. J Endod, 32(3), 2006, 210-3.

[9] Annamalai S, Mungara J. Efficacy of mineral trioxide aggregate as an apical plug in non-vital young permanent teeth: preliminary results. J Clin Pediatr Dent. 35(2), 2010, 149-55. 